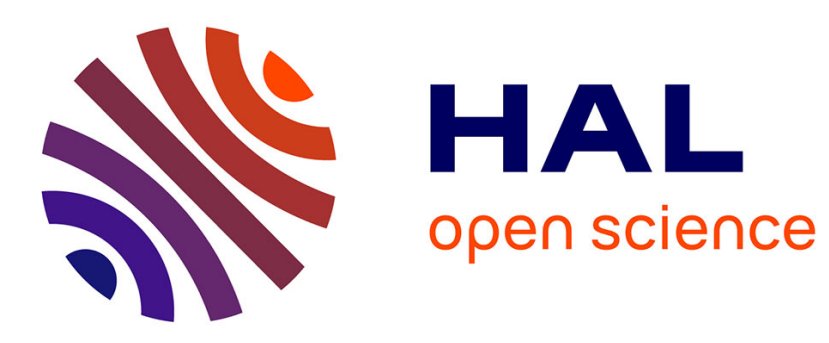

\title{
Visual Behaviour Based Bio-Inspired Polarization Techniques in Computer Vision and Robotics
}

\author{
Abd El Rahman Shabayek, Olivier Morel, David Fofi
}

\section{To cite this version:}

Abd El Rahman Shabayek, Olivier Morel, David Fofi. Visual Behaviour Based Bio-Inspired Polarization Techniques in Computer Vision and Robotics. Developing and Applying BiologicallyInspired Vision Systems: Interdisciplinary Concepts, Information Science Reference, pp.247-276, 2012, 10.4018/978-1-4666-2539-6.ch011 . hal-00760950

\section{HAL Id: hal-00760950 https://hal.science/hal-00760950}

Submitted on 4 Dec 2012

HAL is a multi-disciplinary open access archive for the deposit and dissemination of scientific research documents, whether they are published or not. The documents may come from teaching and research institutions in France or abroad, or from public or private research centers.
L'archive ouverte pluridisciplinaire HAL, est destinée au dépôt et à la diffusion de documents scientifiques de niveau recherche, publiés ou non, émanant des établissements d'enseignement et de recherche français ou étrangers, des laboratoires publics ou privés. 


\title{
Visual Behavior Based Bio-Inspired Polarization Techniques in Computer Vision and Robotics
}

\author{
Abd EI Rahman Shabayek, Olivier Morel, and David Fofi \\ Le2i - UMR CNRS 5158, IUT Le Creusot, Université de Bourgogne, France
}

\begin{abstract}
For long time, it was thought that sensing of polarization in animals is invariably related to their behavior like navigation and orientation. Recently, it was found that polarization can be part of a high-level visual perception, permitting a wide area of vision applications. Polarization vision can be used for most tasks of color vision like: object recognition, contrast enhancement, camouflage breaking, and signal detection and discrimination. The polarization based visual behavior in the animal kingdom will be briefly covered. Then, we will go in depth with the bio-inspired applications based on polarization in computer vision and robotics. The aim is to have a comprehensive survey highlighting the key principles of polarization based techniques and how they are biologically inspired.
\end{abstract}

\section{INTRODUCTION}

Bio-inspiration is an established concept which is developing to meet the needs of many challenges particularly in machine vision applications. Polarization vision is one of the most important biological features in the animal kingdom. A broad range of applications has been inspired from it. So, what is polarization? Polarization is the phenomenon that describes the oscillations orientation of the light (or other radiation) waves which are restricted in direction (Goldstein, 2003). Light's vector orientation can be surprisingly weekly detected by some humans with their naked eyes (Haidinger, 1844), but humans need the help of polarizing optics to visualize most invisible polarization effects (Green, Ohmann, Leininger, \& Kavanaugh, 2010).

Many fish, cephalopods, crustaceans, insects, and other animals are capable of perceiving polarized light (Horváth \& Varjú, 2004). Most animal photoreceptors are able to differentially react to partially linearly polarized light (Goldsmith, 1975; Nilsson \& Warrant, 1999; Waterman, 1981; Wehner, 2001). Fish (Hawryshyn, 1992) and birds (Phillips \& Waldvogel, 1988) photoreceptors respond to polarized light patterns and hence are able to analyze linear polarization (Cronin et al., 2003).

Firstly, we will briefly cover the polarization based visual behavior in the animal kingdom, especially behaviors that can be mapped directly to the machine vision world such as orientation and navigation, water and transparent object detection, camouflage breaking, and communication. Secondly, a comprehensive cover of polarization-inspired machine vision applications will be given. Finally, the future research directions in bio-inspired machine vision applications based on polarization will be discussed. 
The main part of the chapter will go into details regarding bio-inspired polarization techniques in robotics applications. We start with a short survey of how to visualize polarization information. Then a detailed complete survey of robot orientation and navigation techniques based on polarization will be given due to its importance for both communities of computer vision and robotics and its active ongoing research. A comprehensive survey of underwater polarization vision is also given due to the challenging problem of enhancing vision underwater and how it is greatly improved using polarization. Moderate survey of communication (few methods are clearly bio-inspired) and camouflage breaking techniques based on polarization are then covered. Finally, examples of general computer vision techniques based on polarization are mentioned.

Our objective is to give a top view of polarization applications in computer vision and robotics, especially the bio-inspired polarization depended techniques in order to have a comprehensive coverage of such important and active area of research.

\section{POLARIZATION BASED VISUAL BEHAVIOR IN THE ANIMAL KINGDOM}

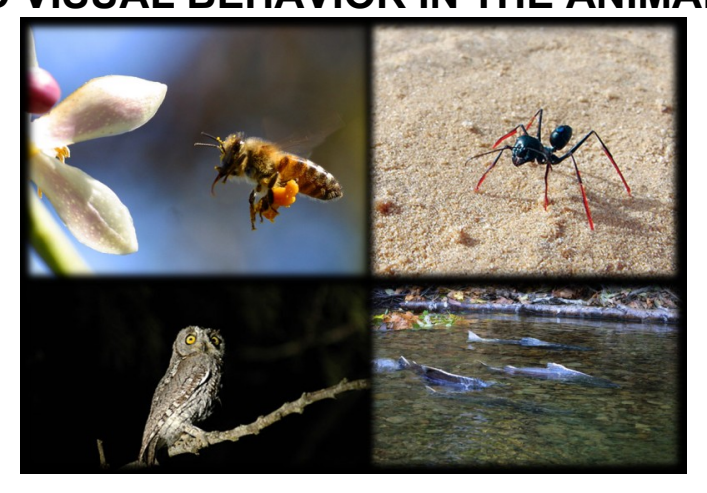

Figure 1: Polarization in the animal kingdom

\section{Orientation and Navigation}

Sky polarization patterns are used by many insects for navigation. Honeybees use celestial polarization to move between the hive and foraging locations (Cronin et al., 2003; Rossel,1989; Wehner, 2001).

Salmon fishes may have a similar ability (Hawryshyn, 1992), which allows them to orient in underwater light fields (Novales \& Hawryshyn, 1997). Light reflection from water surfaces results in horizontally polarized light which is utilized by water beetles and other insects for orientation. (Schwind, 1983; Schwind, 1984; Schwind, 1991).

\section{Water and Transparent Object Detection}

Water surfaces can be discriminated from virtual surfaces (like mirages) by flying insects using their polarization vision ability (Horváth \& Wehner, 1997; Cronin et al., 2003). Reflected polarized light is used by aquatic insects such as dragon- flies, mayflies, and backswimmers to detect calm water surfaces (Horváth \& Varjú 2004). In (Lythgoe \& Hemmings, 1967) they first proposed to enhance the transparent targets visibility in water using polarization. It was found that there are some objects which strongly reflect polarized light underwater (Cronin \& Shashar, 2001; Cronin et al., 2003). It is proved that squids and their relatives see obscure objects using polarized light (Shashar, Adessi, \& Cronin, 1995, Shashar, Milbury, \& Hanlon, 2002).

\section{Camouflage Breaking}

Camouflage is a tool to defeat visual detection by predators. (Lythgoe \& Hemmings, 1967) proposed that polarization could be used to detect well-camouflaged targets in water. Polarization vision helps to 
detect transparent preys (Shashar, Hanlon, \& Petz, 1998). Cephalopods (squid, cuttlefish, and octopus) can produce a variety of body patterns for camouflage using their optically malleable skin that contains neutrally controlled pigmented chromatophores as well as structural light reflectors and some of them are able to see camouflaged objects (Hanlon \& Messenger, 1996; Shashar \& Hanlon, 1997; Shashar et al. 1998; Shashar, Hagan, Boal, \& Hanlon, 2000). In (Mäthger, \& Hanlon, 2006) they give evidence that the polarized aspect of iridescent color in squid skin produces highly dynamically changeable camouflaged patterns.

\section{Communication}

Polarization patterns are used for signaling by some animals. Some of these signals are controlled by the reflection of linearly polarized light. Forests butterflies use their wings as identifying markers by utilizing their polarization-dependent reflectivity (Sweeney, Jiggins, \& Johnsen, 2003; Douglas, Cronin, Chiou, \& Dominy, 2007). Males seem to recognize females based on that (Sweeney et al., 2003; Cronin et al., 2003; Douglas et al., 2007).

Although the spectral irradiance varies strongly underwater with depth, signal constancy is stable and predictable thanks to polarization (Ivanoff \& Waterman, 1958). Linked to specific communications, polarized light is strongly reflected from many stomatopod species body parts that are specialized for that kind of reflection (Cronin et al., 2003). Polarized-light signals and color signals are used in much the same way by mantis shrimps (Cronin et al., 2003). Cephalopods can produce body patterns for signaling using their skin that contains controlled pigmented chromatophores and structural light reflectors (Hanlon \& Messenger, 1996).

\section{POLARIZATION-INSPIRED MACHINE VISION APPLICATIONS}

It is clear that the polarized patterns seen by some animals have an important impact on image formation as they are significant image features within the animals' visual fields. These significant polarized features can be transferred to the computer world leading toward numerous biologically inspired applications in camera technology, image formation, computer vision and robotics. A lot of researchers have already made use of polarization features in nature and inspired artificial vision techniques. Some of the current applications which imitate the animal natural behavior are found in robot orientation and navigation, camouflage breaking, communication, and underwater vision. Scene segmentation and object detection, recognition, and tracking are also active research areas.

There are many applications that utilize polarization but are not biologically inspired (e.g. camera calibration, fiber optics communication, etc). Therefore these methods are out of the scope of this chapter, however, a section will be dedicated to list different examples of polarization based applications in computer vision. This chapter mainly covers methods that imitate animal's behavior.

\section{Visualizing Polarization Information}

Polarization-Difference Imaging (PDI) inspired by polarization vision of certain animal species was introduced by (Rowe, Pugh, Tyo, \& Engheta, 1995). They demonstrated that PDI techniques may facilitate targets detection in scattering media even if targets show very weak polarization or are distant far from the optical setup (Wehner, 1987; Lin, Yemelyanov, Pugh, \& Engheta, 2004; Rowe et al., 1995).

In order to represent polarization without affecting other visual information such as color and brightness, special sensory substitution forms are required. (Yemelyanov, Lo, Pugh, \& Engheta, 2003; Yemelyanov, Lin, Luis, Pugh, \& Engheta, 2003) investigated several bio-inspired representational methodologies for mapping polarization information into visual cues readily perceived by the human visual system. The visual cues and strategies they explored were a) the use of coherently moving dots superimposed on 
image to represent various range of polarization signals, b) overlaying textures with spatial and/or temporal signatures to isolate image regions with differing polarization, c) modulating luminance and/or scenes color contrast in terms of certain aspects of polarization values, and d) fusing polarization images into intensity-only images. They tried to determine which mappings are most suitable for specific applications such as object detection, navigation, sensing, scene classifications, and surface deformation. (Lin et al., 2004) proposed to use these visual cues to enhance visual surveillance techniques using polarization.

\section{Robot Orientation and Navigation}

Many researchers have made theoretical and experimental investigations on skylight polarization for autonomous orientation and navigation. The sky is polarized due to the scattering of sunlight by particles and air molecules in the atmosphere. The sky polarization patterns present us with the polarization information which can be used as an external compass. The location of the sun mainly determines the celestial skylight polarization pattern. It is visible and stable even in open sky patches when the sun is occluded by clouds, and it also appears beneath dark objects in air that is illuminated by the sun (Pomozi, Horváth, \& Wehner, 2001; Suhai \& Horváth, 2004). The benefit of using the skylight polarization pattern, rather than directly using the sun is that only patches of sky are sufficient for orientation task (Cronin, Warrant, \& Greiner, 2006).

Firstly, orientation techniques using photodiodes as a primary sensor to read polarization data will be covered. Secondly, as the camera technology became less expensive and more advanced, new techniques using CCD, CMOS, and/or Fisheye lens to obtain polarization information will be covered. Thirdly, water and mud detection for off-road navigation will be covered. All methods require a rotating polarizer as a primary optic combined with the mentioned sensors as will be explained.

\section{Sensors using Photodiodes}

Inspired by the basic biological neuronal circuit, (Lambrinos et al. 1997; Lambrinos et al. 1998; Möller, Lambrinos, Pfiefer, Labhart, \& Wehner, 1998; Lambrinos, Möller, Labhart, Pfeifer, \& Wehner, 2000) have developed polarization-opponent units (POL- OP units) as input devices that are functionally similar to the POL-neurons found in insects. Each POL-OP unit consists of a pair of polarized light sensors (POL-sensors) followed by a log-ratio amplifier where:

- The POL-sensors consist of photodiodes with a linear polarizer and a blue transmitting filter on the top.

- In each POL-OP unit the polarizing axis of one POL-sensor was adjusted $90^{\circ}$ to the polarizing axis of the other sensor, thus mimicking the crossed-analyzer configuration in the POL-area of insect eyes.

- The signals of each pair of POL-sensors were fed into a log ratio amplifier.

- The three pairs of POL-sensors were mounted on a mobile robot and adjusted such that the polarizing axis of the positive channel was $0^{\circ}, 60^{\circ}$ and $120^{\circ}$ (similar to the insect layout) with respect to the robot's body axis. The visual fields of the POL-OP units are about $60^{\circ}$ and are centered around the zenith.

There are two models to obtain compass direction from the POL-OP responses: a) scanning models and b) simultaneous models. In scanning models (Lambrinos et al. 1997), the agent has to:

1. Find the solar meridian to use it as a reference direction $0^{\circ}$ for its proprioceptive system.

a. For doing that it has to actively scan the sky by rotating around its vertical body axis.

b. When the output signal of one POL-OP unit (or a combination of multiple POL-OP units) reaches its maximum, the robot is known to be aligned with the solar meridian.

2. It uses proprioceptive information to find its heading direction based on the solar meridian. 
3. The compass direction was obtained by comparing the current output values of the POL-OP units with a lookup table that associates the output values of the POL-OP units with the corresponding orientation of the robot.

4. The lookup table should be recorded before each experiment by a single $360^{\circ}$ rotation of the robot.

In contrast, the heading direction can be determined continuously and no scanning movements are necessary in a simultaneous model. In (Lambrinos et al. 2000) they implemented a simultaneous model which does not require a lookup table, but uses an analytical procedure to derive compass information from the values of the POL-OP units. The change in the polarization pattern during the day has to be taken into account. This can be done by either regularly updating the lookup table or by normalizing the outputs of the POL-OP units as follows:

1. The POL-OP signals are delogarithmized by applying a sigmoid function.

2. Find the two candidate orientations (an ambiguity of $\pi$ exists) from the equation derived in (Lambrinos et al. 2000) $\phi=\frac{1}{2} \arctan \frac{p_{1}(\phi)+2 p_{2}(\phi)-\frac{3}{2}}{\sqrt{3}\left(p_{1}(\phi)-\frac{1}{2}\right)}$ where $p_{i}$ is the sensors output.

3. Solve the ambiguity by employing a set of ambient-light sensors on the robot.

The values from eight ambient-light sensors, arranged in two half-circles covering a visual field of $180^{\circ}$, are used to obtain a rough estimate of the robot's heading with respect to the sun (ambient-light sensors with the visual field enclosing the solar meridian will have a stronger response).

4. Transform the current POL-OP readings to signals that are independent of the degree of polarization as shown in (Lambrinos et al. 2000).

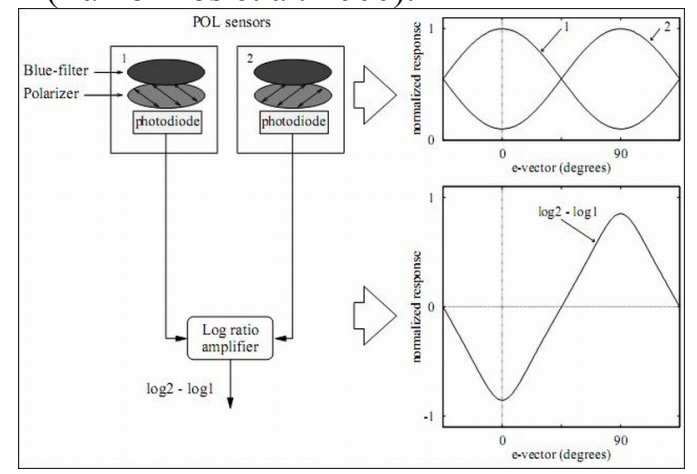

Figure 2: Diagrammatic description of POL OP unit. (Adapted from (Lambrinos et al., 2000; Chu et al., 2007))

In (Chu, Zhao, Zhang, \& Wang, 2007a; Chu, Zhao, Wang \& Zhang, 2007b; Chu, Zhao, Zhang, \& Wang, 2008; Chu, Wang, Chen, \& Li, 2009; Zhao, Chu, Wang, \& Zhang, 2009) they have a bionic navigation sensor which is similar to (Lambrinos et al. 1997; Lambrinos et al. 1998; Möller et al. 1998; Lambrinos et al. 2000) with the same POL-OP unit (see figure (2)) and used the same mathematical formulation. Their design has:

- Three polarization direction analyzers whose polarizing axis of the positive channel is adjusted to $60^{\circ}$ difference from one to one.

- Each one consists of two POL-sensors which have the shape of regular triangular prisms (total of six sensors). 
- For each direction analyzer, the polarizing axis of one POL-sensor was adjusted $90^{\circ}$ to the polarizing axis of the other sensor.

- A POL-OP unit consists of a pair of POL-sensors and a log-ratio amplifier.

○ The log-ratio amplifier receives input from the two POL-sensors and delivers the difference of their logarithmized signals (exactly the same idea technique of Lambrinos et al).

- The three direction analyzers are mounted on a base plate.

- Six ambient-light sensors are arranged in a ring and mounted in the metal cylinder of the six POL-sensors.

- Each ambient-light sensor consists of standard photoresistors with blue filter in front.

- They simulated their design in Simulink ${ }^{\circledR}$ and analyzed the output error. Then some outdoor experiments were carried out.

All outdoor experiments performed by (Lambrinos et al. 1997; Lambrinos et al. 1998; Möller et al. 1998; Lambrinos et al. 2000) and (Chu et al. 2007a; Chu et al. 2007b; Chu et al. 2008; Chu et al. 2009; Zhao et al. 2009) proved to have high accuracy in obtaining directional information from polarization and the error is independent of the traveling distance.

In (Schmolke \& Mallot, 2002) the performance of the path integration without external reference is compared with the performance using a polarization compass. As proprioceptive estimate, wheel revolutions were used in both cases. The experiments were carried out using a Khepera miniature robot. They found that the e-vector compass was much better in accuracy.

In (Chahl \& Mizutani, 2010) they developed two biomimetic sensors and made flight tests for stabilization and navigation of an aircraft using the spatial, polarization, and spectral distribution of light in the environment. They tried to mimic the head of a dragonfly called Hemianax Papuensis. Here we consider the polarization sensor only:

- It used three photodiodes each with their own optics and polarization filters.

$\circ$ The output of the photodiode amplifiers were digitized and processed on a microcontroller.

- Each photodiode had a voltage bias and gain that required calibration.

- They considered the direction computation from three samples from an assembly of diode, filter, and amplifier at known angles $\left(0^{\circ}, 60^{\circ}\right.$, and $\left.120^{\circ}\right)$ relative to the orientation of the polarization axis of the polarization filter.

The response of each photodetector to incident light in terms of voltage $v$ is $v=b+P . F+q$, which includes the response to unpolarized light $q$ and a bias term $b$ which is due to the electronics. The response of the sensor to the polarized component of the light is given by P.F where $F$ is a vector representing the orientation of the polarization axis of the filter and $P$ is a vector representing the direction and magnitude of the incident polarized light. To eliminate electronic and optical biases ( $b$ and $q$ ) the difference between the responses of the three samples $\mathrm{v}_{1}$ to $\mathrm{v}_{3}$ was taken. Solving for $P$ gives:

$$
P^{T}=\left[\begin{array}{l}
v_{1}-v_{2} \\
v_{1}-v_{3}
\end{array}\right]\left[F_{1}-F_{2} F_{1}-F_{3}\right]^{-1} \text { where } F_{1}-F_{2} \text { is a column vector. }
$$

This solution assumes a calibrated system. To test their sensor, a remotely piloted aircraft was instrumented with a calibrated polarimeter and attitude reference that included a magnetic compass. The flight was run early in the morning when the sun was low. The polarization compass produced a discontinuity several times during the flight as the solution passed through $180^{\circ}$. There were minor differences between the two measures. They conclude that the absence of any correction for attitude probably contributed to most deviation between the magnetic and north-aligned polarization heading. 


\section{CCD, Fisheye and CMOS Sensors}

In (Usher, Ridley, \& Corke, 2001) they proposed to perceive the sky polarization pattern using a color CCD video camera and a linear polarizing film as a filter. The blue component was used for analysis as the sunlight polarization is most apparent at UV and blue wavelengthes (350-450 nm). They smoothed all images by a 2D Gaussian function to overcome the poor response at these short wavelengthes. Two images were taken at a time, with the second image having its polarizing filter axis orthogonal to the first. Taking a set of images, they modeled the response as $f(\Phi)=K[1+d \cos (2 \Phi)]$ where $K$ is a scaling factor dependent on camera shutter settings and ambient conditions, $d$ is degree of polarization, $\Phi$ is the orientation of the polarizing filter with respect to the solar meridian (line connection the zenith and the sun), and $f(\Phi)$ is the mean intensity of an image. Their model had the same form for the photosensitive diodes of (Lambrinos et al. 2000). Their initial experiments proved that it is possible to locate the solar meridian using a digital camera applying the scanning method of (Lambrinos et al. 1997) and hence extracting a reference bearing from an arbitrary orientation.

The mentioned methods are simulating insect strategy taking advantage of the skylight polarization through single numerical values rather than patterns. In (Wu, Gao, Fan, \& Xie, 2010) they presented a method to get navigation orientations by gradient vectors of skylight polarization maps even if the sun is invisible or occluded by clouds. The maps were provided by a zenith centered imaging polarimeter with narrow field of view. The imaging polarimeter was constructed by a sensitive industrial CCD camera with short focus lens and a linearly polarizing filter. Assisted by a compass and gradienter, the imaging polarimeter was kept horizontal with the local sea level so its field of view centre was adjusted to the zenith of the sky area during the experiments. Then the linear polarizer mounted in front of the objective lens was rotated. For a certain sky area, four images were taken by rotating the linear polarizer to four different relative positions $\left(0^{\circ}, 45^{\circ}, 90^{\circ}\right.$, and $\left.135^{\circ}\right)$ following the method in (Lee, 1998). They estimated the solar azimuth by searching the maximum attenuation gradient vector among different azimuth relative to zenith in the degree of polarization (DOP) map, or the symmetrical axis in the angle of polarization (AOP) map making use of the attenuation from zenith to horizon along the local meridian in the DOP maps and the symmetrical distribution of AOP along the local meridian. They concluded that the skylight polarization maps are able to supply stable solar azimuths information and if aided by calendar (insect circadian clock), the real body orientation could be located.

In (Sarkar, Bello, Hoof, \& Theuwissen, 2010a; Sarkar, Bello, Hoof, \& Theuwissen, 2010b; Sarkar, Bello, Hoof, \& Theuwissen, 2010c; Sarkar, Bello, Hoof, \& Theuwissen, 2010d) a polarization analyzing CMOS image sensor was proposed. It is able to sense polarization information in real time using a metallic wire grid micro-polarizer oriented in various directions on top of the pixel. The DOP and AOP can be computed using three intensity images $\left(0^{\circ}, 45^{\circ}\right.$, and $\left.90^{\circ}\right)$ using the Stokes parameters as in (Malacara, 1989; Gerhart \& Matchko, 2002; Damask, 2005), the variations of which can be used as compass clues. The image sensor consist of an array of $128 \times 128$ pixels, occupies an area of $5 \times 4 \mathrm{~mm} 2$ and it has been designed and fabricated in a $180 \mathrm{~nm}$ CMOS process. They concluded to use the computed polarization information as a clue for autonomous robot navigation.

Unlike previous investigations, (Miyazaki, Ammar, Kawakami, \& Ikeuchi, 2009) analyzed sky polarization patterns with the fisheye lens. They have tilted the measurement system based on a fisheye lens, a CCD camera, and a linear polarizer, in order to analyze transition of the $180^{\circ}$ sky polarization patterns while tilting. The used technique is similar to the technique described in (Pomozi et al. 2001; Hegedüs, Åkesson, \& Horváth, 2007). Three photographs were taken for three different alignments $\left(0^{\circ}\right.$, $45^{\circ}$, and $90^{\circ}$ ) of the transmission axis of the polarizer clockwise from the top view of the camera. Their main contribution was to analyze the sky polarization patterns when the camera was tilted from the horizon. They presented a method to determine the solar meridian orientation from photos taken outdoors, 
while only a small part of the sky was available and the camera sensor was not horizontal. Therefore, the orientation of the camera could be determined from the solar meridian.

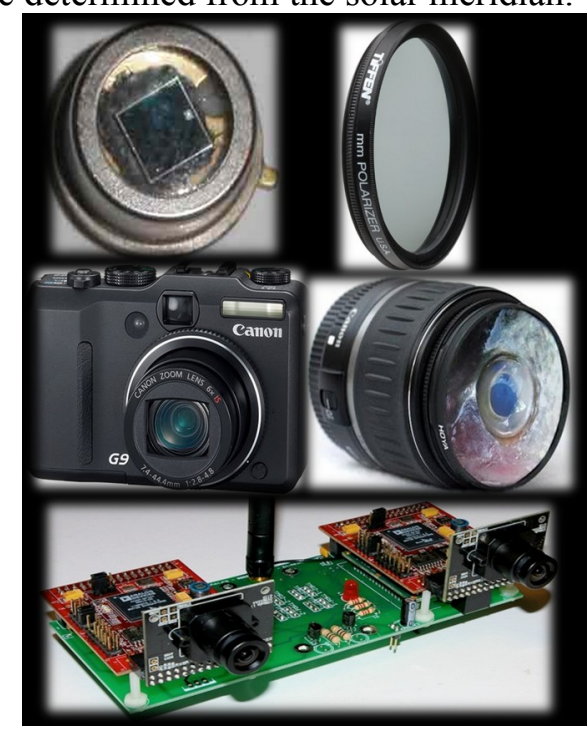

Figure 3: Different visual sensors

\section{Water and mud detection for off-road navigation}

Water and mud detection based on polarization depends on the physical principle that the light reflected from water surface is partially linearly polarized and the polarization phases of them are more similar than those from the scenes around. These hazards can be detected by comparison of polarization degree and similarity of the polarization phases. There is a comparison between different approaches of water and mud hazards detection in (Matthies, Bellutta, \& Mchenry, 2003; Rankin \& Matthies, 2008) and a survey in (Iqbal, Morel, \& Meriaudeau, 2010).

Icy or wet roads present dangerous situations as there is an increased danger of losing control of the vehicle and the glare from wet roadway may hide path markings and other road features. A polarizing filter can considerably reduce reflections due to the fact that water tends to horizontally polarize reflected light. In (Huber et al., 1997) they have developed a spectro-polarimetric imager. It is a portable machine vision system that operates at video frame rates. It contains only electronically controllable components, including an imaging acousto-optic tunable filter, a phase retarder, and a standard CCD-based camera. The system operates much like an ordinary CCD camera, except that the spectral and polarimetric content of light to be viewed is electronically controlled using computer. During operation, the host computer sends commands to the controller to select the desired spectral and polarization parameters. They proposed glare reduction and glare detection in which their imaging system could be applied to wet or icy road conditions. Glare reduction works similarly to a polarizing filter inserted in the optical path. However, the phase retarder accomplishes this electronically and can be programmatically enabled and disabled, which is beneficial since an additional filter reduces the overall intensity of the image. For glare detection, they identify horizontally polarized reflections, thereby detecting possible dangerous road conditions. In a computer-assisted driving scenario, this additional information could be used to alert the driver of the danger, or in an autonomous vehicle, the controller could modify its driving habits accordingly.

In (Yamada, Ueda, Horiba, \& Sugie, 2001) to discriminate wet road, the ratio between horizontal and vertical polarization image intensity for each pixel was used. This algorithm obtained good discrimination accuracy when applied to highway environment where most water reflects the sky. A drawback of this 
polarization ratio based method is that it becomes imprecise when water reflects other aspects of the environment, which is common in off-road navigation.

In (Sarwal, Nett, \& Simon, 2004) they have made use of two approaches for small water bodies' detection. The first one requires use of an existing custom camera with three polarization filters $\left(0^{\circ}, 45^{\circ}\right.$, and $90^{\circ}$ ); intrinsically mounted such that these filters and connected optics view exactly the same scene. The other approach requires use of three physically distinct cameras with the same type of polarization filters mounted on three low-cost cameras each with similar optics, running with certain geometric approximations due to the flat-earth assumption. There are pros and cons for each approach. Both approaches used the formulas from (Wolff, 1995).

In order to detect water hazards for autonomous off-road navigation, especially bodies of water which roofed with tree canopy, (Xie, Xiang, Pan, \& Liu, 2007) presented another polarization method based on the similarity between polarization patterns reflected from water surface than those from other scenes. Their detection algorithm is based on the comparison of DOP and similarity of the AOP using the formulas from (Wolff, 1995).

In (Pandian, 2008) he used polarization imaging and stereo vision to detect water hazards for robot navigation. He investigated the conventional single camera polarization imaging setup employing a mechanically rotated polarizer, polarization contrast imaging using two cameras, a three camera setup enabling the complete characterization of partially linearly polarized light, and a four camera setup employing two polarizers with identical orientations. The main challenge in their proposed system was to improve the pixel correspondence across the polarization images. They obtained good results for water detection, however further in-depth experiments are still required.

Robust mud detection is also a critical perception requirement for autonomous navigation. Mud is highly polarized and hence polarization based techniques can be used. In (Rankin, \& Matthies, 2008) they proposed to use multiple sensors for mud detection including a polarization camera. At a pixel level, partial linear polarization is measured by the transmitted radiance through a polarization filter. To determine the polarization state (orientation, intensity, and DOP), three images of a scene are acquired, each with the polarization filter at a different orientation. To calculate "polarization contrast" as a simplified measurement, only two images are required where the polarization filter orientations differ by $90^{\circ}$. The "polarization contrast" at each pixel can be calculated by dividing the absolute value of the difference between the $0^{\circ}$ and $90^{\circ}$ intensity values by the sum of them. Regions that have a significantly higher DOP can be a potential cue for water or mud. In the experiments of (Rankin, \& Matthies, 2008) they used two polarization sensors: a SAMBA polarization camera and a SALSA linear stokes polarization camera. The SAMBA camera provides a "polarization contrast" image and the SALSA camera provides DOP, intensity, and orientation images.

\section{Underwater Polarization Vision}

Underwater imaging is widely used in scientific research and technology. Computer vision methods are used for a variety of applications, such as swimming pool life guards, mine detection, inspection of underwater power and telecommunication cables, pipelines, nuclear reactors, research in marine biology, archaeology and mapping.

The underwater polarized light distribution is mainly affected by a) the position of the sun or the moon in the sky, b) the water optical properties, c) the viewing depth, and d) surfaces reflections (Waterman, 1988; Novales \& Hawryshyn, 1997; Cronin \& Shashar, 2001; Shashar, Sabbah, \& Cronin, 2004). There are two distinct polarization patterns underwater, one inside Snell's window (underwater natural lighting comes from a limited cone above the scene which is caused by the illuminating rays refraction through the water surface, and is called the optical manhole or Snell's window) and one outside it. Generally, the 
polarization pattern inside Snell's window in a few meters depths is assumed to be determined by the same factors as those influencing the sky polarization (Waterman, 1988). In (Cronin \& Shashar, 2001) they found only small differences between the polarization patterns within Snell's window and outside it. With increasing depth, the polarization pattern simplifies rapidly, tending to become horizontal everywhere (Timofeeva, 1969; Shashar et al., 2004).

(Lerner, Sabbah, Erlick, \& Shashar, 2010) found that, only in clear waters, the polarization is correlated with the sun's elevation and the maximum value of the e-vector orientation angle equals the angle of refraction in the horizontal viewing direction. They concluded that navigation by means of underwater polarization is possible under restricted conditions like being in clear waters, mainly near the horizontal viewing direction, and in locations where the sea floor has limited effects on the light's polarization. In underwater vision, the scattering of light largely degrades contrast between the observer and any object observed (Lythgoe \& Hemmings, 1967; Wehner, 2001). As a result of the existing horizontal polarization, a vertical analyzer would reduce the scattered light amount perceived and, hence, increase contrast.

In order to improve airborne underwater target detection, a proper polarization filter should be selected. This reduces line of sight problems and false alarms. Detection processing of a polarization filter that gives mostly surface related detections can be used to filter the final detection list as proposed in (Crosby $\&$ Stetson, 2002). They found that reducing the effect of the surface reflections is possible using a linear horizontal and a linear vertical polarizer. One of them contains surface reflection and one does not. If the system is active, the choice is the image from the polarizer that aligns with the light source. Otherwise (if passive), the linear horizontal polarizer image contains the most surface effects. They chose an algorithm called FX target detection (Crosby, \& Riley, 2001) along with the linear polarizers to improve the detection of underwater targets.

The SHallow water Real-time IMagIng Polarimeter (SHRIMP), developed at the Office of Naval Research in 2000, can measure underwater partial polarization imagery (Taylor, \& Wolff, 2001). This sensor is a passive, three-channel device that concurrently measures the three Stokes vector components needed to find out the partial linear polarization of the scene. The testing of this sensor was completed in 2002 and the data was analyzed in (Taylor, Davis, \& Wolff, 2002; Taylor, Davis, \& Wolff, 2003). They presented performance results which showed high probability for detecting the target with a low probability of a false alarm. The tests were done in very shallow water and surf zone regions. Their results support that a) the passive polarization signature is a range dependent quantity that decreases with increased range which do not impact performance greatly, and it is reflective (it is a function of target shape, surface characteristics, and light source level, b) the polarization magnitude signature is higher for the more turbid water.

In (Shashar et al., 2004) they studied how polarization signals vary when seen from different distances in water. To check how polarization changes as a function of distance in water, a polarization target was created. The target was set at different locations and types of water (clear and turbid). The target was videotaped over a range of distances with a custom-built underwater imaging polarimeter where the polarizer was rotated automatically at $\left(0^{\circ}, 45^{\circ}\right.$, and $\left.90^{\circ}\right)$. Images were analyzed based on (Wolff \& Andreou, 1995). Based on these measurements, (Shashar et al., 2004) expect that polarization sensitivity will be most useful in a few meters distance for visual tasks in water. It will be much less useful for detecting objects, signals, or structures from far away. Celestial polarization patterns for navigation and orientation are expected to be limited to shallow waters, while solar navigation is possible through a deeper range.

In order to improve underwater visibility, some methods applied image enhancement either by showing the DOP (Rowe et al., 1995; Taylor \& wolff 2001) or showing the polarization contrast by subtracting 
two orthogonal polarization images (Denes, Gottlieb, Kaminsky, \& Metes, 1998; Harsdorf, Reuter, \& Tönebön, 1999). The previous solutions assumed that polarization is associated with the object radiation, rather than the causes which degrade this signal. This assumption is not valid with increasing distance (Morgan, Khong, \& Somekh, 1997). In natural illumination, underwater polarization is associated with the visibility disturbance (Können, 1985). The approach in (Schechner \& Karpel, 2004; Schechner \& Karpel, 2005; Treibitz \& Schechner, 2009) uses the captured polarization components using an underwater polarimetric camera (Karpel \& Schechner, 2004; Treibitz \& Schechner, 2009) to delete that disturbance and inverts the physical model to recover an image similar to the clear visibility appearance. In their methods, they use two images maintaining the same viewing position while varying the polarization filter orientation. (Sarafraz, Negahdaripour \& Schechner, 2009) integrate polarization analysis with stereovision by extending the single-camera method in (Treibitz \& Schechner, 2006) to a pair of cameras displaced by a finite base line where each camera makes use of a different polarization setting. The stereo disparity and polarization analysis are used to construct enhanced de-scattered views.

In (Li \& Wang, 2009) they propose a method for backscatter rejection where the difference between object and background depolarization characteristics is used to improve image contrast. If the object and the background differ considerably in their depolarization properties, the signal-to-noise ratio (SNR) of the image may increase. (Li \& Wang, 2010) propose a polarization image fusion for the intensity (first Stokes component), DOP, and AOP in a false RGB image to enhance the underwater image contrast.

\section{Communication}

From a communications perspective, polarization modulation of an optical signal is an old concept that was proposed in (Niblack, \& Wolf, 1964). A basic theoretical treatment of a polarization modulation system can be found in (Pratt, 1969; Benedetto \& Poggiolini, 1992). The use of light polarization for sending data is common in fiber optic systems (Chow, Kwok, Liu, Tsang, \& Chinlon, 2006), and has previously been proposed for free space communications (Uehara, Seto, Ohtsuki, Sasase, \& Mori, 1992), but is rarely implemented due to concerns about depolarization in free space communications (Pun, Chan, $\&$ Chen, 2005). Intensity modulation schemes are usually implemented and the polarization of the signal is usually ignored. A comparison of intensity and polarization modulation can be found in (Grosinger, 2008) which proves that polarization modulation outperforms intensity modulation.

Due to the seawater high conductivity which limits the electromagnetic waves propagation, radio frequency communications in seawater are impractical. Current methods, such as acoustic communication, are limited in bandwidth, data rate, and have a high latency (Heidemann, Ye, Wills, Syed, \& Li, 2006). The use of cables is impractical for autonomous vehicles. Optical wireless communications that utilize the blue/green transparency window of seawater offer high bandwidth short range communications (Hanson, \& Radic, 2008).

One useful feature of optical communications is the ability to exploit the light beam polarization to differentiate the received signal from backscatter, other transmitters, or surface light. (Cox, Hughes, \& Muth, 2009) demonstrate a simple and low-cost system to explore the light polarization for underwater communication. The system uses diode lasers and is portable. The system consists of a transmitter and a receiver. The transmitter uses two diode lasers mounted to the faces of a polarizing beam splitter (PBS) to combine the orthogonal polarizations. The receiver is constructed using another PBS with two detectors mounted to the faces which detect the imposed modulated data on the orthogonal polarizations. Their work is based on (Cox, Simpson, Domizioli, Muth, \& Hughes, 2008).

In (Furukawa \& Sakamoto 2010) the authors proposed a communication method using an invisible polarization code which is drawn on the eyes of the robot. A special graphics display for showing expression of robot's eyes was developed. The mobile robots are able to send and receive data, using the invisible polarization code, in order to establish an environment for cooperative robots. The code is a 
variation of QR Code which is a kind of 2D symbology developed by Denso Wave in 1994. QR Code contains information in both the vertical and horizontal directions. This QR Code was used to establish the communication between the mobile robots. To embed the invisible polarization code within the displayed data, they used a polarized symbol image to overlap additional data on the display. Their display consists of a conventional LCD panel, an additional liquid crystal (LC) layer and some optics. The LC layers can rotate the direction of the polarization axis according to the applied voltage. The LC layer in the LCD panel is responsible for showing visual data. The additional LC layer changes the direction of polarization from LCD outputs to generate invisible symbol patterns which are horizontal or vertical linear polarized light waves. This difference of orientation creates a binary symbol image. The invisible code can be perceived using a polarizer.

\section{Camouflage Breaking}

Polarization has a strong role to play in camouflage breaking specially in remote sensing. Camouflage technology has exposed the conventional remote sensing drawback because the natural background makes the targets detection very complicated. Scattering light polarimetric characteristics of camouflaged targets are very different from that of natural backgrounds. Polarimetric imaging can remove the backgrounds influence and improve the inspection efficiency. Compared with reflectance images, polarization images have advantages in camouflage targets detection. Polarimetric imaging has proven to be the most effective approach as it strongly reveals camouflaged targets embedded in complex backgrounds (Egan, 1992; Brun, Jeune, Cariou, \& Lotrian, 1992; Weidemann, Fournier, Forand, \& Mathieu, 2005; Zhang, Cheng, Chen, \& Zheng, 2009a).

In different remote sensing applications, polarimetric techniques were studied to find the optimum polarization for rain cancellation (Tang \& Rubin, 1972), to enhance the image visibility of ocean waves (Schuler, Lee, \& Hoppel, 1993), and to find optimum polarizations in the bistatic scattering from layered random media (Lee \& Mudaliar, 1994). (Dehmollaian \& Sarabandi, 2009) tried to find an optimum polarization set that is able to enhance the signal-to-clutter ratio of a foliage-camouflaged stationary target. They employed a genetic algorithm (GA) to find optimum polarization configurations which minimize the effect of forests on the backscattered radar cross section response of a camouflaged hard target.

A field study of polarimetric characteristics was done by many researchers. It indicated that polarimetric imaging in hot infrared can eliminate the influence of background and enhance the efficiency of detection. (Goldstein, 2000) tested military coatings on aluminous plate. (Egan \& Duggin, 2000; Egan \& Duggin, 2002) studied the aircrafts and camouflaged vehicles polarimetric characteristics. (Forssell \& Karlsson, 2003) carried out polarimetric experiments on vehicles and (Aron \& Gronau, 2005) did it on tents.

In order to set up a portable polarimetric camera, (Cronin, Shashar, \& Wolff, 1994) placed two twisted nematic liquid crystals and a fixed polarizing filter in front of a CCD camera. They presented two configurations: an autonomous sensor that uses a small camcorder for recording images; and an on-line sensor that uses a digital camera connected to a personal. For the same purpose (Zhang et al., 2009a) used a multispectral CCD polarization camera, a trisection prism to divide the scattering light from targets into three parts, and a polarizer. The simplest polarimetric camera may consist of a rotating linear polarizer and a CCD camera.

(Cronin et al., 1994) used their polarimetric camera to break color camouflage. An orange caterpillar camouflaged on an orange leaf show a case where polarization can be used where the orientation of polarization difference is significantly larger than the differences that appear in the brightness of the natural full colored image. Moreover, the AOP variation in the light reflected from the caterpillar is much larger than that reflected from the leaf. 
In (Zhang et al., 2009a) they studied the properties of camouflage targets polarization scattering according to the change of incidence angle and observational angle. They concluded that the spectra of camouflage targets can simulate that of backgrounds. Polarization remote sensing proved that the camouflage targets with the same spectra most likely have very different polarimetric characteristics with background. Camouflage targets can be identified clearly in DOP imaging and AOP imaging. The camouflage effect is lost when it comes from traditional remote sensing to polarization remote sensing.

The spectral scattering light polarization signature is affected by the incidence angle, azimuth and the characteristics of materials. In (Zhang, Cheng, Chen, \& Zheng, 2009b) the coating DOP was studied, and the results indicated that the coating DOP represented a reverse trend with the surface reflectance and azimuth angle. A model based on Fresnel reflection equation was set up to describe the coatings polarization scattering behavior which can be predicted by the model up to visible light and infrared wavelengths.

\section{Miscellaneous}

Polarization filtered images analysis proved to be useful for computer vision. Hence, in this section we will cover different applications in computer vision based on polarization, which are not necessarily bio-inspired, to give the flavor of polarization in this important research area. For example, it was used to:

- $\quad$ analyze speculiarities (Wolff, 1989; Nayar, Fang, \& Boult, 1997; Saito, Sato, Ikeuchi, \& Kashiwagi, (1999a; 1999b)),

- $\quad$ separate transparent and semi-reflected scenes (Farid, \& Adelson, 1999; Schechner, Shamir, \& Kiryati, $(1999 ; 2000))$,

- classify materials (Wolff, 1997; Sarkar2010b),

- catadioptric camera calibration and 3D reconstruction (Morel, Stolz, \& Gorria, 2004a ; Morel, Meriaudeau, Stolz, \& Gorria, 2005; Atkinson, \& Hancock, 2005; Morel, Ferraton, Stolz, \& Gorria, 2006; Morel, Stolz, Meriaudeau, \& Gorria, 2006; Morel, \& Fofi, 2007; Morel, Seulin, \& Fofi, (2007a; 2007b; 2008); Shabayek, Morel, \& Fofi, (2009; 2010); Shabayek, 2010),

- industrial inspection (Morel, Stolz, \& Gorria, 2004b; Morel, Stolz, Meriaudeau, \& Gorria, 2005; Morel, Stolz, \& Gorria, 2006; Meriaudeau, Ferraton, Stolz, Bigué, \& Morel, 2008),

- segmenting scenes (Ben-Ezra2000; Ahmad, \& Takakura, 2007; Terrier, Devlaminck, \& Charbois, 2008; Subramaniam, \& Hancock, 2010; Shabayek, Demonceaux, Morel, \& Fofi, 2011),

- dehazing (Schechner, Narasimhan, \& Nayar, (2001; 2003; 2008)),

- and polarimetric cameras (Shutov, 1993; Cronin et al., 1994; Wolff, Mancini, Pouliquen, \& Andreou, 1997; Ben-Ezra, 2000; Zhang et al., 2009a) which enable acquisition of polarization information in real time to be applied in any of the polarized based machine vision applications.

\section{Summary}

This section will summarize the different areas of bio-inspired polarization based computer vision and robotics applications, highlighting the important points in each area.

Visualizing polarization information: A short survey was given on available methods to visualize polarization information in order to map it into visual cues such as polarization-difference imaging, coherently moving dots superimposed on image, overlaying textures with spatial and/or temporal signatures, modulating luminance and/or scenes color contrast in terms of certain aspects of polarization measured values, and fusing polarization images into intensity-only images. 
Robot orientation and navigation: We surveyed the different bio-inspired polarization techniques for robot orientation and navigation. Photodiodes, $\mathrm{CCD} / \mathrm{CMOS}$, and special sensors were used. The methods using photodiodes are cheap however they are simulating insects' strategy taking advantage of the skylight polarization through single numerical values rather than patterns. The techniques based on $\mathrm{CCD} / \mathrm{CMOS}$ sensors are more expensive but they can analyze the sky polarization patterns giving polarization maps (DOP, AOP, polarization contrast) in order to obtain various orientation and navigation clues. Using a fisheye lens with a CCD sensor is a good solution to obtain an omni-directional polarization image up to $180^{\circ}$. CMOS based techniques are promising for miniature robots as size is important for practical situations however the viewing angle is limited. Stereo vision based techniques provide different points of view but require more equipment and they are not practical especially if the cameras do not share the same baseline. Polarimetric cameras are a good solution as they acquire the polarization information in one shot which is better than conventional sensors which need at least three acquisitions. The spectro-polarimetric imager is good in the sense that it is possible to obtain both polarimetric and spectral information to be fused to obtain various clues for orientation and navigation.

Underwater polarization vision: The effect of water surface reflections can be reduced using a linear horizontal and a linear vertical polarizer. The passive polarization signature decreases with increased range, and it is a function of target shape, surface characteristics, and light source level. Polarization sensitivity will be most useful in a few meters distance for visual tasks in water. In short distances, it is valid to assume that polarization is associated with the object radiation, and hence it is possible to use DOP, AOP, and DPI to enhance the image. However it is not valid with long distance, therefore, the visibility disturbance should be deleted and the physical model of the disturbance cause should be inverted to obtain a clear image.

Communication: Polarization modulation outperforms intensity modulation. Optical communications are able to exploit the light beam polarization to differentiate the received signal from any other signal in air and underwater. It is possible to show invisible polarization code on a display (e.g robot's eyes) to be embedded within visual information. Polarization information can be used effectively for cooperative robots.

Camouflage breaking: Polarization has a strong role to play in camouflage breaking specially in remote sensing. Polarimetric imaging has proven to be the most effective approach as it strongly reveals camouflaged targets embedded in complex backgrounds. The camouflage targets with the same spectra most likely have very different polarimetric characteristics with background. The camouflage effect can be lost when it comes from traditional sensing to polarization sensing.

Miscellaneous: Polarization filtered images analysis proved to be useful for computer vision and robotics applications. It has been used in a broad range of applications like specularities analysis, separating transparent and semi-reflected scenes, material classification, camera calibration, 3D reconstruction, industrial inspection, scene segmentation, de-hazing, and developing new camera technologies (e.g polarimetric cameras).

\section{FUTURE RESEARCH DIRECTIONS}

We think that there are three important fields of polarization research which will evolve in the next few years: spectral-polarization vision, omni-polarization vision, and a combination of both (omni-spectro-polarization vision).

Independently, polarimetric and spectral features do not completely represent an object of interest (Zhao, Gong, \& Pan, 2008). The objects elemental composition defines the reflected spectral signature. The polarization characteristics depend on surface features, such as smoothness and conductance. These 
features can be combined to reduce false alarms, improve target identification, and enhance the scene description. Some work has already been done on this combination in (Giakos, 2006; Zhao, Pan, \& Zhang, 2006; Zhao et al., 2008; Zhao, Zhang, L., Zhang, D. \& Pan, 2009; Cummings et al., 2009).

Omni-directional polarization vision is required to perceive the surrounding $360^{\circ}$ polarization features. Omni-vision can be obtained by wide angle lenses, fisheye lens, multi-cameras, and catadioptric sensors (a combination of mirror and lens). Catadioptric sensors provide the best omni-vision view as it is able to capture the complete surrounding which is up to $360^{\circ}$ with minimal occlusions. Omni-directional polarization vision has been established by combining a fisheye lens and polarization filter in (Miyazaki et al., 2009). It has been also proposed as a combination of special designed mirror placed into a cylindrical polarization filter in (Horita et al., 2008; Horita, Shibata, Maeda, \& Hayashi, 2009). Another design based on cone mirror and CCD camera for surveillance in (Lin et al., 2004) equipped with a linear/circular polarizer. They capture three images at $\left(0^{\circ}, 45^{\circ}\right.$, and $\left.90^{\circ}\right)$. However, in (p.220) they wrongly stated that "For reflective mirror with metallic reflection surface, however, the effects on polarization are simpler. For many highly reflective metallic surfaces the polarization of reflected light is the mirror image of the original polarization pattern." The light polarization state is changed after being reflected from metallic surfaces (e.g a linearly polarized light reflects as an elliptically polarized light) (Born \& Wolf, 1999, p.741). This problem has been solved by calibrating the original incident light polarization state from the reflected state in (Shabayek, Morel, \& Fofi, 2011a).

The future of polarization vision is going to be omni-directional due to its wide range of applications and the amount of information captured at one location and one shot. It takes the conventional vision applications into the omni-directional dimension. To overcome the special design, and multiple captures (at least three) requirements, a novel omni-polarization catadioptric sensor is proposed in the next section.

Finally, we propose to have an omni-spectro-polarimetric camera (e.g combining a mirror with FD-1665 3CCD Multispectral Cameras with a linear polarizer at 0, 60, 120 in front of each camera configured by the manufacturer). It is inspired by the birds' field of view which can reach $360^{\circ}$, spectral vision of the Daphnia magna, and polarization vision of Cataglyphis ants. The proposed sensor will be able to show the spectral and polarimetric features from an omni-directional window. It means that we will be able to sense $360^{\circ}$ of the surrounding environment at one shot. Imagine the power of such sensor! It can be used in almost all machine vision applications enhancing the quality of perceived images and fusing comprehensive information in a single image. It may greatly enhance the autonomous robotics world especially in open environments. To the best of our knowledge, here is the first time to propose such sensor.

\section{Non-Central Omni-Polarization Catadioptric Sensor}

We propose to use a non-central omni-polarization catadioptric sensor composed of a mirror and a polarimetric camera at $\left(0^{\circ}, 60^{\circ}\right.$, and $\left.120^{\circ}\right)$ as an optimum combination (Tyo, 1998). It can be used for most of the machine vision applications. We have recently proposed to use it for sky/ground segmentation for unmanned air vehicle (UAV) attitude estimation (Shabayek, Demonceaux, Morel, \& Fofi, 2011).

Polarization information are directly computed from three intensity images taken at three different angles $\left(0^{\circ}, 45^{\circ}\right.$, and $\left.90^{\circ}\right)$ or $\left(0^{\circ}, 60^{\circ}\right.$, and $\left.120^{\circ}\right)$ at one shot using a polarimetric camera. The polarization patterns consist of the AOP $\phi$ and the DOP $\rho$ are defined as:

$$
\phi=\arctan \left(\frac{S_{2}}{S_{1}}\right), \rho=\frac{\sqrt{S_{1}^{2}+S_{2}^{2}}}{S_{0}}
$$

where $S_{i}$ are called the Stokes components (Goldstein, 2003) can be computed from: 


$$
I_{0}=0.5\left(S_{0}+S_{1} \cos (2 \alpha)+S_{2} \sin (2 \alpha)\right.
$$

where $\alpha$ is the polarizer angle. Hence:

$$
\begin{gathered}
S_{0}=I_{0}+I_{90}, S_{1}=I_{0}-I_{90}, S_{2}=2 I_{45}-I_{0}-I_{90}, \text { or } \\
S_{0}=\frac{I_{60}}{2}+\frac{I_{120}}{2}+I_{0}, S_{1}=\frac{I_{60}}{2}-\frac{I_{120}}{2}+I_{0}, S_{2}=\frac{2}{\sqrt{3}}\left(\frac{I_{60}}{2}-\frac{3 * I_{120}}{2}+3 * I_{0}\right)
\end{gathered}
$$

where $I_{0}, I_{45}$, and $I_{90}$ are intensity images taken at $\left(0^{\circ}, 45^{\circ}\right.$, and $\left.90^{\circ}\right)$ or $I_{00}$, $I_{60}$, and $I_{120}$ are intensity images taken at $\left(0^{\circ}, 60^{\circ}\right.$, and $\left.120^{\circ}\right)$ at one shot using a polarimetric camera (e.g FluxData FD-1665 series like FD-1665P-M which has three monochrome CCDs with 0, 45, 90 or 0, 60, 120 linear polarizers) .

Figure (4) shows the segmentation results for non-central catadioptric images with the horizon detected by detecting the polarization transition areas. Unlike conventional segmentation methods, thanks to polarization, the illumination problem caused by the sun is solved in the image domain.

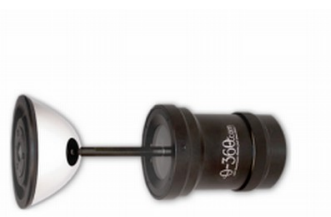

(a) Catadioptric sensor

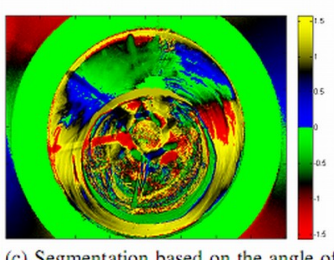

(c) Segmentation based on the angle of polarization

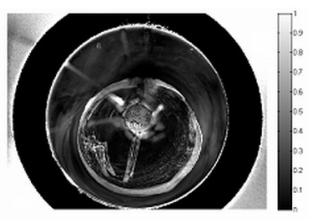

(b) Segmentation based on the de gree of polarization

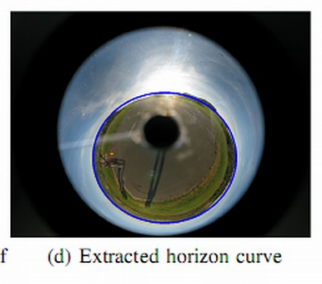

Figure 4: Sky/Ground segmentation and horizon extraction based on polarization from non-central catadioptric images

The sky polarization patterns can be obtained using the proposed sensor, however in order to use it the original incident polarization state should be calibrated as the reflected light from the metallic mirror has a different polarization state from the incident light (Born \& Wolf, 1999, p.741). Once the pattern is obtained the sun azimuth and zenith angle can be computed. Given a built in calendar in the autonomous robot with the extracted sun information, the orientation information can be obtained. Figure (5) shows a simulation of the sky polarization pattern in Le Creusot, France at 16:00 on 15-Mar-2011 using the proposed omni-pola-catadioptric sensor. This work still under progress and there are unpublished promising results (Shabayek, Morel, \& Fofi, 2011b).

We believe that polarization information have strong cues for orientation and navigation. In the near future it will be possible to use the sky polarization patterns in night for robot autonomous navigation like day light using the moon polarization patterns inspired by the nocturnal (Dacke, Byrne, Baird, Scholtz, \& Warrant, 2011). 


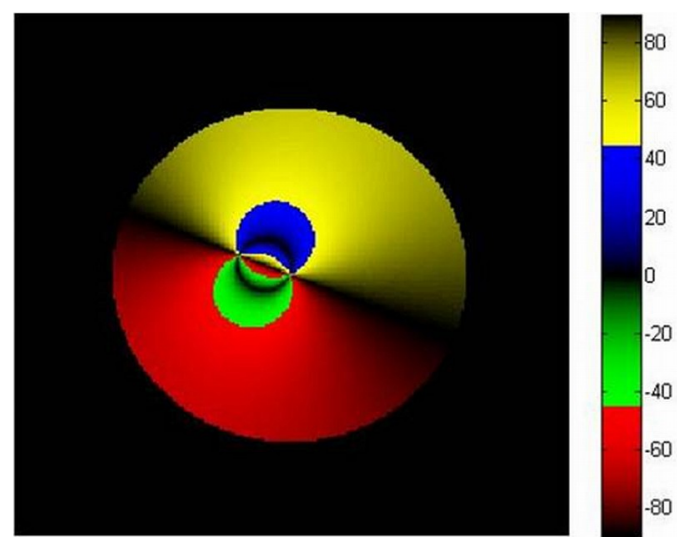

Figure 5: Simulation of the sky angle of polarization pattern in Le Creusot, France at 16:00 on 15-Mar-2011 using a pola-catadioptric sensor.

\section{CONCLUSION}

Animal's behavior is extremely flexible and robust facing environmental contingencies. By adopting some of these behaviors in machines, it is possible to obtain similar flexibility and robustness. Many aspects of animals lead to biological inspiration like a) behavioral strategies, b) physical design, and c) the nervous systems organization. In this chapter, we focused on the animals' polarization based visual behavior strategies.

Human visual awareness can be greatly expanded by augmenting different sensor models by the different capabilities of computer vision systems. Polarization vision represented in polarimetric cameras becomes available to a broad range of audience, specifically outdoors and underwater applications. Thanks to polarimetric cameras, it will be easier to have real time bio-inspired robotics. Unmanned robots for surveillance or exploring tasks may greatly benefit from such sensors.

Polarization information has strong cues for orientation and navigation. It can be used efficiently in communication, remove backscattering for underwater vision, break camouflage in complex backgrounds, and adapted in various machine vision applications where analyzed polarization filtered images proved to be useful.

A complete dedicated survey to one of the most important bio-inspired visual features in nowadays applications was presented, and future views of possible sensors were proposed.

\section{REFERENCES}

Ahmad, J. E. \& Takakura, Y. (2007). Improving segmentation maps using polarization imaging. In IEEE International Conference on Image Processing, Vol. 1 (pp. I -281 -I -284).

Aron, Y. \& Gronau., Y. (2005). Polarization in the LWIR. In Proceedings of SPIE, Vol. 5783 (pp. 653-661).

Atkinson, G. A. \& Hancock, E. R. (2005). Multi-view surface reconstruction using polarization. In Proceedings of the IEEE International Conference on Computer Vision.

Benedetto, S. \& Poggiolini, P. (1992). Theory of polarization shift keying modulation. IEEE Transactions on Communications 40, 708-721. 
Ben-Ezra, M. (2000). Segmentation with invisible keying signal. In Proceedings of IEEE Conference on Computer Vision and Pattern Recognition (pp. 32-37).

Born, M. \& Wolf, E. (1999). Principles of optics: Electromagnetic Theory of Propagation, Interference and Diffraction of Light (7th Edition). Cambridge University Press.

Brun, G. L., L.Jeune, B., Cariou, J. \& Lotrian, J. (1992). Analysis of polarization signature of immersed targets. In Proceedings of SPIE Polarization and Remote Sensing, Vol. 1747 (pp. 128-139).

Chahl, J. \& Mizutani, A. (2010). Biomimetic attitude and orientation sensors. Sensors Journal, IEEE PP (99), 1.

Chow, C. W., Kwok, C. H., Liu, Y., Tsang, H. K. \& Chinlon, L. (2006). 3-bit/symbol optical modulation-demodulation simultaneously using DRZ, DPSK and PolSK. In Conference on Lasers and Electro-Optics (CLEO).

Chu, J., Zhao, K., Zhang, Q. \& Wang, T. (2007a). Design of a novel polarization sensor for navigation. In IEEE International Conference on Mechatronics and Automation.

Chu, J., Zhao, K., Wang, T. \& Zhang, Q. (2007b). Research on a novel Polarization sensor for navigation. In IEEE International Conference on Information Acquisition. .

Chu, J., Zhao, K., Zhang, Q. \& Wang, T. (2008). Construction and performance test of a novel polarization sensor for navigation. Sensors and Actuators A: Physical 148, 75-82.

Chu, J., Wang, H., Chen, W. \& Li, R. (2009). Application of a novel polarization sensor to mobile robot navigation. In Mechatronics and Automation, 2009. ICMA 2009. International Conference on (pp. 3763 -3768).

Cox, W. C., Simpson, J., Domizioli, C. P., Muth, J. \& Hughes, B. (2008). An underwater optical communication system implementing Reed-Solomon channel coding. In Proceedings of OCEANS Conference.

Cox, W. C., Hughes, B. L. \& Muth, J. F. (2009). A polarization shift-keying system for underwater optical communications. In OCEANS 2009, MTS/IEEE Biloxi - Marine Technology for Our Future: Global and Local Challenges.

Cronin, T. W., Shashar, N. \& Wolff, L. B. (1994). Portable imaging polarimeters. In Pattern Recognition, 1994. Vol. 1 - Conference A: Computer Vision Image Processing., Proceedings of the 12th IAPR International Conference, Vol. 1 (pp. 606 -609).

Cronin, T. W. \& Shashar, N. (2001). The linearly polarized light field in clear, tropical marine waters: spatial and temporal variation of light intensity, degree of polarization, and e-vector angle. J. Exp. Biol 204, 24612467.

Cronin, T. W., Shashar, N., Caldwell, R. L., Marshall, J., Cheroske, A. G. \& Chiou, T.-H. (2003). Polarization vision and its role in biological signaling. Integrative and Comparative Biology 43 (4), 549-558. 
Cronin, T. W., Warrant, E. J. \& Greiner, B. (2006). Celestial polarization patterns during twilight. Optical Society of America. Applied Optics 45.

Crosby, F. \& Riley, S. (2001). Signature Adaptive mine detection at a constant false alarm rate. In Proceeding of SPIE Conference on Detection and Remediation Technologies for Mines and Minelike Targets.

Crosby, F. \& Stetson, S. (2002). Surface effect subtraction for airborne underwater target detection. In OCEANS MTS/IEEE.

Cummings, M. E., Ahmed, S. A., Dierssen, H. M., Gilerson, A., Gilly, W. F., Kattawar, G. W., Seibel, B. A. \& Sullivan, J. M. (2009). Biological response to the dynamic spectral-polarized underwater light field. TEXAS UNIVERISITY AT AUSTIN.

Dacke, M., Byrne, M. J., Baird, E., Scholtz, C. H. \& Warrant, E. (2011). How dim is dim? Precision of the celestial compass in moonlight and sunlight. Philosophical Transactions of The Royal Society.

Biological Sciences B 366, 697-702.

Damask, J. N. (Ed.). (2005). Polarization optics in telecommunications, Vol. 101. Springer.

Dehmollaian, M. \& Sarabandi, K. (2009). Optimum polarizations for discrimination of a Foliage-camouflaged target, using genetic algorithms. IEEE Geoscience and Remote Sensing Letters 6 (1), $82-86$.

Denes, L. J., Gottlieb, M., Kaminsky, B. \& Metes, P. (1998). AOTF polarization difference imaging. SPIE 3584, 106-115.

Douglas, J. M., Cronin, T. W., Chiou, T.-H. \& Dominy, N. J. (2007). Light habitats and the role of polarized iridescence in the sensory ecology of neotropical nymphalid butterflies (Lepidoptera: Nymphalidae). Journal of Experimental Biology 210, 788-799.

Egan, W. G. (1992). Polarization in remote sensing. In Proc. SPIE 1747. Elsevier.

Egan, W. G. \& Duggin, M. J. (2000). Optical enhancement of aircraft detection using polarization. In Proceedings of SPIE, Vol. 4133 (pp. 172-178).

Egan, W. G. \& Duggin, M. J. (2002). Synthesis of optical polarization signatures of military aircraft. In Proceedings of SPIE, Vol. 4481 (pp. 188-194). .

Farid, H. \& Adelson, E. H. (1999). Separating reflections and lighting using independent components analysis. In Proceedings of IEEE Conference on Computer Vision and Pattern Recognition (pp. 262-267).

Forssell, G. \& Karlsson, E. H. (2003). Measurements of polarization properties of camouflaged objects and of the denial of surfaces covered with cenospheres. In Proceedings of SPIE, Vol. 5075 (pp. 246-258).

Furukawa, T. \& Sakamoto, K. (2010). Eye contact communication system between mobile robots using invisible code display. In H. Yang, R. Malaka, J. Hoshino \& J. Han (ed.),Entertainment Computing ICEC 2010, Vol. 6243 (pp. 468-471). Springer Berlin / Heidelberg.

Gerhart, G. \& Matchko, R. (2002). Visualization techniques for four Stokes parameter polarization. In Proc. of the ground target modeling and validation conference (pp. 216-225). 
Giakos, G. C. (2006). Multifusion, multispectral, optical polarimetric imaging sensing principles. IEEE Transactions on Instrumentation and Measurement 55, 1628-1633.

Goldsmith, T. (1975). The polarization sensitivity-dichroic absorption paradox in arthropod photoreceptors. Photoreceptor optics, Springer Verlag, Berlin. A. W. Snyder and R. Menzel (eds.), 98-125.

Goldstein, D. H. (2000). Polarimetric characterization of federal standard paints. In Proceedings of SPIE, Vol. 4133 (pp. 112-123).

Goldstein, D. (2003). Polarized Light, Revised and Expanded (Optical Science and Engineering). CRC.

Green, A. S., Ohmann, P. R., Leininger, N. E. \& Kavanaugh, J. A. (2010). Polarization imaging and insect vision. The Physics Teacher 48 (1), 17-20.

Grosinger, J. (2008). Investigation of polarization modulation in optical free space communications through the atmosphere. Unpublished master's thesis, Institut fur Nachrichtentechnik und Hochfrequenztechnik, eingereicht an der Technischen Universitat Wien, Fakultat fur Elektrotechnik und Informationstechnik.

Hanlon, R.T. \& Messenger, J.B. (1996). Cephalopod behaviour. Cambridge, UK: Cambridge University Press.

Hanson, F. \& Radic, S. (2008). High bandwidth underwater optical communication. Applied Optics 47, 277-283.

Harsdorf, S., Reuter, R. \& Tönebön, S. (1999). Contrast-enhanced optical imaging of submersible targets. SPIE 3821, 378-383.

Hawryshyn, C. (1992). Polarization vision in fish. American Scientist 80, 164-175.

Hegedüs, R., Åkesson, S. \& Horváth, G. (2007). Polarization patterns of thick clouds: overcast skies have distribution of the angle of polarization similar to that of clear skies. J. Opt. Soc. Am. 24, 2347-2356.

Heidemann, J., Ye, W., Wills, J., Syed, A. \& Li, Y. (2006). Research challenges and applications for underwater sensor networking. In In Proceedings of the IEEE Wireless Communications and Networking Conference (pp. 228--235).

Haidinger, W. (1844). Über das directe Erkennen des polarisirten Lichts und der Lage der Polarisationsebene.. Poggendorfs Annalen 63, 29-39.

Horita, Y., Hayashi, Y., Shibata, K., Hayashi, K., Morohashi, K. \& Doi, T. (2008). Omni-directional polarization image capture using omni-directional camera and polarization filter. In ITST 2008. 8th International Conference on ITS Telecommunications, 2008. (pp. 99 -102). .

Horita, Y., Shibata, K., Maeda, K. \& Hayashi, Y. (2009). Omni-directional polarization image sensor based on an omni-directional camera and a polarization filter. In IEEE International Conference on Advanced Video and Signal Based Surveillance (pp. 280 -285). 
Horváth, G., Gál, J. \& Wehner, R. (1997). Why are water-seeking insects not attracted by mirages? The polarization properties of mirages. Naturwissenschaften $\mathbf{8 4}, 300-303$.

Horváth, G. \& Varjú, D. (2004). Polarized light in animal vision: Polarization patterns in nature. Springer.

Huber, D. F., Denes, L., Hebert, M., Gottlieb, M., Kaminsky, B. \& Metes, P. (1997). A Spectro-polarimetric imager for intelligent transportation systems. In SPIE International Symposium on Intelligent Systems and Advanced Manufacturing, Intelligent Transportation Systems, Vol. 3207.

Iqbal, M., Morel, O. \& Meriaudeau, F. (2010). A survey on outdoor water hazard detection. In The 5th International Conference on Information \& Communication Technology and Systems.

Ivanoff, A. \& Waterman, T. H. (1958). Factors, mainly depth and wavelength, affecting the degree of underwater light polarization. J. Mar. Res 16, 283-307.

Karpel, N. \& Schechner, Y. Y. (2004). Portable polarimetric underwater imaging system with a linear response. In Proc. SPIE Polarization: Measurement, Analysis and Remote Sensing VI.

Können, G. P. (1985). Polarized light in nature. Cambridge University Press.

Lambrinos, D., Maris, M., Kobayashi, H., Labhart, T., Pfeifer, R. \& Wehner, R. (1997). An autonomous agent navigation with a polarized light compass. Adaptive Behaviour 6, 131-161.

Lambrinos, D., Maris, M., Kobayashil, H., Labhart, T., Pfeifer, R. \& Wehner, R. (1998). Navigating with a polarized light compass. In Self-Learning Robots II: Bio-robotics (Digest No. 1998/248), IEE (pp. 7/1 $-7 / 4)$.

Lambrinos, D., Möller, R., Labhart, T., Pfeifer, R. \& Wehner, R. (2000). A mobile robot employing insect strategies for navigation. Robotics and Autonomous Systems 30, 39-64.

Lee, J. K. \& Mudaliar, S. (1994). Optimum polarizations in the bistatic scattering from layered random media. IEEE Transactions on Geoscience and Remote Sensing 32, 169-176.

Lee, R. L. (1998). Digital imaging of clear-sky polarization. Applied Optics 37, 1465-1476.

Lerner, A., Sabbah, S., Erlick, C. \& Shashar, N. (2010). Navigation by light polarization in clear and turbid waters. Philosophical Transactions of The Royal Society Biological Sciences 366, 671-679.

Li, Y. \& Wang, S. (2009). Underwater polarization imaging technology. In Conference on Lasers Electro Optics The Pacific Rim.

Li, Y. \& Wang, S. (2010). Underwater object detection technology based on polarization image fusion. In 5th International Symposium on Advanced Optical Manufacturing and Testing Technologies: Optoelectronic Materials and Devices for Detector, Imager, Display, and Energy Conversion Technology, Proc. of SPIE.

Lin, S.-S., Yemelyanov, K. M., Pugh, J. E. N. \& Engheta, N. (2004). Polarization enhanced visual surveillance techniques. In Proceedings of the IEEE International Conference on Networking. Sensing \& Control. 
Lythgoe, J. N. \& Hemmings., C. C. (1967). Polarized light and underwater vision. Nature 213, 893-894.

Malacara, D. (1989). Physical optics and light measurements. In Academic Press (pp. 157).

Matthies, L., Bellutta, P. \& Mchenry, M. (2003). Detecting water hazards for autonomous off-road navigation. In Proceedings of SPIE Conference 5083: Unmanned Ground Vehicle Technology V (pp. 263-352).

Meriaudeau, F., Ferraton, M., Stolz, C., Bigué, L. \& Morel, O. (2008). Polarization imaging for industrial inspection. In SPIE Electronic Imaging - Machine Vision Applications in Industrial Inspection XIII, Vol. 6813.

Miyazaki, D., Ammar, M., Kawakami, R. \& Ikeuchi, K. (2009). Estimating sunlight polarization using a fish-eye lens. IPSJ Transactions on Computer Vision and Applications 1, 288-300.

Morel, O., Ferraton, M., Stolz, C. \& Gorria, P. (2006). Active lighting applied to shape from polarization. In IEEE International Conference on Image Processing. (pp. 2181-2184).

Morel, O. \& Fofi, D. (2007). Calibration of catadioptric sensors by polarization imaging. In IEEE International Conference on Robotics and Automation.

Morel, O., Meriaudeau, F., Stolz, C. \& Gorria, P. (2005). Polarization imaging applied to 3D reconstruction of specular metallic surfaces. In SPIE Electronic Imaging - Machine Vision Applications in Industrial Inspection XIII, Electronic Imaging, Vol. 5679 (pp. 178-186). .

Morel, O., Seulin, R. \& Fofi, D. (2007a). Measurement of the Three-dimensional mirror parameters by polarization imaging applied to catadioptric camera calibration. In IEEE/SPIE 8th International Conference on Quality Control by Artificial Vision (QCAV'2007).

Morel, O., Seulin, R. \& Fofi, D. (2007b). Catadioptric camera calibration by polarization imaging. In Iberian Conference on Pattern Recognition and Image Analysis.

Morel, O., Seulin, R. \& Fofi, D. (2008). Measurement of three-dimensional mirror parameters by polarization imaging applied to catadioptric camera calibration. Journal of Electronic Imaging 17 (3).

Morel, O., Stolz, C. \& Gorria, P. (2006). Polarization imaging for 3D inspection of highly reflective metallic objects. Optics and Spectroscopy 101 (1), 15-21.

Morel, O., Stolz, C. \& Gorria, P. (2004a). Polarization applied to 3D reconstruction of highly reflective metallic objects. In $O S A V$.

Morel, O., Stolz, C. \& Gorria, P. (2004b). Application of polarimetric imaging to 3D inspection of highly reflective metallic surface. In SPIE Optics East - Two- and Three-Dimensionale Vision Systems for Inspection, Control, and Metrology II, Vol. 5606 (pp. 82-89). .

Morel, O., Stolz, C., Meriaudeau, F. \& Gorria, P. (2005). Three-dimensional inspection of highly-reflective metallic objects by polarization imaging. Electronic Imaging Newsletter 15 (2), 4.

Morel, O., Stolz, C., Meriaudeau, F. \& Gorria, P. (2006). Active lighting applied to 3D reconstruction of specular metallic surfaces by polarization imaging. Applied Optics 45 (17), 4062-4068. 
Morgan, S. P., Khong, M. P. \& Somekh, M. G. (1997). Effects of polarization state and scatterer concentration on optical imaging through scattering media. Applied Optics 36, 1560-1565.

Mäthger, L. M. \& Hanlon, R. T. (2006). Anatomical basis for camouflaged polarized light communication in squid. Biology letters 2(4), 494-496.

Möller, R., Lambrinos, D., Pfiefer, R., Labhart, T. \& Wehner, R. (1998). Modeling ant navigation with an autonomous agent. In Proceedings of the fifth international conference on simulation of adaptive behavior on From animals to animats.

Nayar, S. K., Fang, X. S. \& Boult, T. (1997). Separation of reflection components using color and polarization. International Journal of Computer Vision 21, 163-186.

Niblack, W. \& Wolf, E. (1964). Polarization modulation and demodulation of light. Applied Optics 3, 277-277.

Nilsson, D.-E. \& Warrant, E. J. (1999). Visual discrimination: Seeing the third quality of light. Curr. Biol, 9R535-R537. 9, 535-537.

Novales, F. I. \& Hawryshyn., C. W. (1997). Is the use of underwater polarized light by fishes restricted to crepuscular time periods?. Vision Res 37, 975-989.

Phillips, J. B. \& Waldvogel, J. A. (1988). Celestial polarized light patterns as a calibration reference for sun compass of homing pigeons. Journal of Theoretical Biology 131, 55-67.

Pomozi, I., Horváth, G. \& Wehner, R. (2001). How the clear-sky angle of polarization pattern continues underneath clouds: full-sky measurements and implications for animal orientation. The Journal of Experimental Biology 204, 2933-2942.

Pratt, W. K. (1969). Laser communication systems. New York: Wiley.

Pun, S., Chan, C. \& Chen, L. (2005). A novel optical frequency shift keying transmitter based on polarization modulation. In Optical Fiber Communication Conference. Technical Digest. OFC/NFOEC, Vol. 3 .

Rankin, A. \& Matthies, L. (2008). Daytime mud detection for unmanned ground vehicle autonomous navigation. In Proceedings of the 26th Army Science Conference. .

Rossel, S. (1989). Polarization sensitivity in compound eyes. Facets of vision, Springer Verlag, Berlin In D. G. Stavenga and R. C. Hardie (eds.), 298-316.

Rowe, M. P., Pugh, E. N., Tyo, J. S. \& Engheta, N. (1995). Polarization-difference imaging: a biologically inspired technique for observation through scattering media. Optics Letters 20, 608-610.

Sarafraz, A., Negahdaripour, S. \& Schechner, Y. Y. (2009). Enhancing images in scattering media utilizing stereovision and polarization. In Proceedings of IEEE Workshop on Applications of Computer Vision.

Sarkar, M., Bello, D. S. S., Hoof, C. V. \& Theuwissen, A. (2010a). Biologically inspired autonomous agent navigation using an integrated polarization analyzing CMOS image sensor. Procedia Engineering $5,673-676$. 
Sarkar, M., Bello, D. S. S., Hoof, C. V. \& Theuwissen, A. (2010b). Integrated polarization-analyzing CMOS image sensor for detecting incoming light ray direction. In Sensors Applications Symposium, 2010 IEEE (pp. 194 -199). .

Sarkar, M., Bello, D. S. S., Hoof, C. V. \& Theuwissen, A. (2010c). Integrated polarization-analyzing CMOS image sensor. In Proceedings of 2010 IEEE International Symposium on Circuits and Systems (pp. $621-624)$.

Sarkar, M., Bello, D. S. S., Hoof, C. V. \& Theuwissen, A. (2010d). Integrated polarization analyzing CMOS Image sensor for autonomus navigation using polarized light. In IEEE Conf. of Intelligent Systems (pp. 224-229). .

Sarwal, A., Nett, J. \& Simon, D. (2004). Detection of small water-bodies. PercepTek Robotics 12395 N. Mead Way Littleton, CO 80125.

Saito, M., Sato, Y., Ikeuchi, K. \& Kashiwagi, H. (1999a). Measurement of surface orientations of transparent objects using polarization in highlight. Journal of the Optical Society of America 16(9), 2286 2293.

Saito, M., Sato, Y., Ikeuchi, K. \& Kashiwagi, H. (1999b). Measurement of surface orientations of transparent objects using polarization in highlight. In Proceedings of IEEE Conference on Computer Vision and Pattern Recognition.

Schechner, Y. Y. \& Karpel, N. (2004). Clear underwater vision. In IEEE Conference on Computer Vision and Pattern Recognition (pp. 536 543).

Schechner, Y. Y. \& Karpel, N. (2005). Recovery of underwater visibility and structure by polarization analysis. IEEE Journal of Oceanic Engineering 30, 631-636.

Schechner, Y. Y., Narasimhan, S. G. \& Nayar, S. K. (2001). Instant dehazing of images using polarization. In Proceedings of IEEE Conference on Computer Vision and Pattern Recognition (pp. 325-332).

Schechner, Y. Y., Narasimhan, S. G. \& Nayar, S. K. (2003). Polarization-based vision through haze. Applied Optics 42, 511-525.

Schechner, Y. Y., Narasimhan, S. G. \& Nayar, S. K. (2008). Polarization-based vision through haze. In Proceeding of ACM SIGGRAPH ASIA, Vol. 42.

Schechner, Y. Y., Shamir, J. \& Kiryati, N. (1999). Polarization-based decorrelation of transparent layers: The inclination angle of an invisible surface. In Proceedings of IEEE International Conference on Computer Vision (pp. 814-819).

Schechner, Y. Y., Shamir, J. \& Kiryati, N. (2000). Polarization and statistical analysis of scenes containing a semi-reflector. Journal of the Optical Society of America A 17, 276-284.

Schuler, D. L., Lee, J. S. \& Hoppel, K. W. (1993). Polarimetric SAR image signatures of the ocean and Gulf Stream features. IEEE Transactions on Geoscience and Remote Sensing 31, 1210-1221. 
Schwind, R. (1984). The plunge reaction of the backswimmer Notonecta glauca.. Journal of Comparative Physiology A, 155, 319-321.

Schwind, R. (1983). Zonation of the optical environment and zonation in the rhabdom structure within the eye of the backswimmer, Notonecta glauca. Cell and Tissue Research 232, 53-63.

Schwind, R. (1991). Polarization vision in water insects and insects living on a moist substrate. Journal of Comparative Physiology A, 169, 531-540.

Shabayek, A. E. R., Fofi, D. \& Morel, O. (2009). A novel non-central catadioptric auto-calibration approach. In 9th Workshop on Omnidirectional Vision (OMNIVIS) in conjunction with International Conference on Computer Vision (ICCV).

Shabayek, A. E. R., Morel, O. \& Fofi, D. (2010). Auto-calibration and 3D reconstruction with non-central catadioptric sensors using polarization imaging. In 10th Workshop on Omnidirectional Vision (OMNIVIS) in conjunction with Robotics Systems and Science RSS.

Shabayek, A. E. R. (2010). Non-central catadioptric sensors auto-calibration. LAP Lambert Academic Publishing.

Shabayek, A. E. R., Demonceaux, C., Morel, O. \& Fofi, D. (2011). Vision based UAV attitude estimation: Progress and insights. ICUAS'11 - International Conference on Unmanned Aircraft Systems, a special issue in Journal of Intelligent and Robotic Systems, Accepted.

Shabayek, A. E. R., Morel, O. \& Fofi, D. (2011a). Polarization in the eye of a catadioptric Sensor. unpublished work.

Shabayek, A. E. R., Morel, O. \& Fofi, D. (2011b). Polarization as seen by a catadioptric sensor: A simulation and calibration toolbox. unpublished work.

Shashar, N., Adessi, L. \& Cronin., T. W. (1995). Polarization vision as a mechanism for detection of transparent objects. Ultraviolet radiation and coral reefs, HIMB Tech D. Gulko and P. L. Jokiel (eds.), 207211.

Shashar, N. \& Hanlon., R. (1997). Squids (Loligo pealii and Euprymna scolopes) can exhibit polarized light patterns produced by their skin. The Biological Bulletin 193, 207-208.

Shashar, N., Hanlon, R. \& Petz., deM. A. (1998). Polarization vision helps detect transparent prey. Nature 393, 222-223.

Shashar, N., Hagan, R., Boal, J. G. \& Hanlon, R. T. (2000). Cuttlefish use polarization sensitivity in predation on silvery fish. Vision Res 40, 71-75.

Shashar, N., Milbury, C. \& Hanlon, R. (2002). Polarization vision in cephalopods: Neuroanatomical and behavioral features that illustrate aspects of form and function. Marine and Freshwater Behaviour and Physiology 35, 5768.

Shashar, N., Sabbah, S. \& Cronin, T. W. (2004). Transmission of linearly polarized light in seawater: implications for polarization signaling. Journal of Experimental Biology 207, 3619-3628.

Shutov, A. M. (1993). Videopolarimeters. Soviet Journal of Optical Technology 60, 295-301. 
Schmolke, A. \& Mallot, H. A. (2002). Polarisation compass for robot navigation. In D. Polani, J. Kim, \& T. Martinez (eds.) Fifth German Workshop on Artificial Life. .

Subramaniam, N. \& Hancock, E. (2010). Surface material segmentation using polarisation. Lecture Notes in Computer Science 6256, 115-124.

Suhai, B. \& Horváth, G. (2004). How well does the Rayleigh model describe the E-vector distribution of skylight in clear and cloudy conditions? A full-sky polarimetric study. Optical Society of America A 21, 1669-1676.

Sweeney, A., Jiggins, C. \& Johnsen, S. (2003). Insect communication: Polarized light as a butterfly mating signal. Nature 423, 31-32.

Tang, C. H. \& Rubin, M. D. (1972). Optimum polarization for rain cancellation. In Proceedings of IEEE Antennas and Propagation Society International Symposium, Vol. 10.

Taylor, J.S., J. \& Wolff, L. (2001). Partial polarization signature results from the field testing of the SHallow water Real-time IMaging polarimeter (SHRIMP). In OCEANS MTS/IEEE Conference and Exhibition.

Taylor, J.S., J., Davis, P. \& Wolff, L. (2002). Underwater partial polarization signatures from the SHallow water Real-time IMaging Polarimeter (SHRIMP). In OCEANS MTS/IEEE.

Taylor, J.S., J., Davis, P. \& Wolff, L. (2003). Underwater partial polarization signatures from the shallow water real-time imaging polarimeter (SHRIMP). In Spie proceedings, Detection and Remediation Technologies for Mines and Minelike Targets VIII.

Terrier, P., Devlaminck, V. \& Charbois, J. M. (2008). Segmentation of rough surfaces using a polarization imaging system. Optical Society of America 25 (2), 423-430.

Timofeeva, V. A. (1969). Plane of vibrations of polarized light in turbid media. Izvestiya Atmos. Ocean. Physics 5, 603-607.

Treibitz, T. \& Schechner, Y. Y. (2006). Instant 3Descatter. In Proc. IEEE Computer vision and pattern recognision.

Treibitz, T. \& Schechner, Y. Y. (2009). Active polarization descattering. IEEE Transactionson pattern analysis and machine intelligence 31, 385-399.

Tyo, J. S., Rowe, M. P., Pugh, E. N., Jr. \& Engheta, N. (1996). Target detection in optically scattered media by polarization-difference imaging. Applied Optics 35, 1855-1870.

Tyo, J. S. (1998). Optimum linear combination strategy for an N-channel polarization-sensitive imaging or vision system. Journal of Optical Society 15, 359-366.

Uehara, H., Seto, I., Ohtsuki, T., Sasase, I. \& Mori, S. (1992). Phase noise insensitive multilevel POLSK based on QAM mapping in coherent optical systems. In Singapore ICCS/ISITA. Communications on the Move. 
Usher, K., Ridley, P. \& Corke, P. (2001). A Camera as a Polarized Light Compass: Preliminary Experiments. In Australian Conference on Robotics and Automation. .

Waterman, T. H. (1981). Polarization sensitivity. H. Autrum (ed.), Handbook of sensory physiology, Springer Verlag, Berlin. VII/6B, 281-463.

Wehner, R. (1987). Matched filters' -neural models of the external world. Journal of Comparative Physiology A 161, 511-531.

Wehner, R. (2001). Polarization vision a uniform sensory capacity?. Journal of Experimental Biology 204, 2589-2596.

Weidemann, A., Fournier, G. R., Forand, L. \& Mathieu, P. (2005). In harbor underwater threat detection/ identification using active imaging. In Proceedings of SPIE, Photonics for Port and Harbor Security, Vol. 5780 (pp. 59-70).

Wolff, L. B. (1989). Using polarization to separate reflection components. In Proceedings of IEEE Conference on Computer Vision and Pattern Recognition (pp. 363-369).

Wolff, L. B. (1995). Applications of polarization camera technology. IEEE Expert 10 (5), 30 -38.

Wolff, L. B. \& Andreou, A. G. (1995). Polarization camera sensors. Image and Vision Computing 13, 497-510.

Wolff, L., Mancini, T., Pouliquen, P. \& Andreou, A. (1997). Liquid crystal polarization camera. Robotics and Automation, IEEE Transactions on 13 (2), 195 -203.

Wolff, L. B. (1997). Polarization vision: a new sensory approach to image understanding. Image and Vision Computing 15, 81-93.

Wu, L., Gao, J., Fan, Z. \& Xie, Z. (2010). How to get navigation information within patches of sky as insects do? A primitive orientation by skylight polarization maps. In 2nd International Conference on Signal Processing Systems.

Xie, B., Xiang, Z., Pan, H. \& Liu, J. (2007). Polarization-based water hazards detection for autonomous off-road navigation. In IEEE/RSJ International Conference on Intelligent Robots and Systems.

Yamada, M., Ueda, K., Horiba, I. \& Sugie, N. (2001). Discrimination of the road condition toward understanding of vehicle driving environments. IEEE Transaction on Intelligent Transportation Systems 2(1), $26-31$.

Yemelyanov, K. M., Lo, M. A., Pugh, J. E. N. \& Engheta, N. (2003). Display of polarization information by coherently moving dots. Optics Express 11, 1577-1584.

Yemelyanov, K. M., Lin, S.-S., Luis, W. Q., Pugh, J. E. N. \& Engheta, N. (2003). Bio-inspired display of polarization information using selected visual cues. In Proceedings of SPIE - The International Society for Optical Engineering 5158.

Zhang, Z. Y., Cheng, H. F., Chen, Z. H. \& Zheng, W. W. (2009a). Analysis of polarimetric characteristics of camouflage targets. In Proceedings of SPIE 4th International Symposium on Advanced 
Optical Manufacturing and Testing Technologies: Optical Test and Measurement Technology and Equipment, Vol. 7283.

Zhang, Z. Y., Cheng, H. F., Chen, Z. H. \& Zheng, W. W. (2009b). Analysis of polarimetric scattering for backgrounds and camouflage materials. In Proceedings of SPIE International Symposium on Photoelectronic Detection and Imaging: Advances in Infrared Imaging and Applications, Vol. 7383. .

Zhao, Y.-Q., Gong, P. \& Pan, Q. (2008). Object detection by Spectropolarimeteric imagery fusion. IEEE Transactions on Geoscience and Remote Sensing 46, 3337-3345.

Zhao, Y., Pan, Q. \& Zhang, H. (2006). Object detection by fusion spectral and polarimetric imagery. In Proceedings of SPIE Vol. 6031. .

Zhao, K., Chu, J., Wang, T. \& Zhang, Q. (2009). A Novel angle algorithm of polarization sensor for navigation. IEEE Transactions on Instrumentation and Measurement 58, 2791-2796.

Zhao, Y., Zhang, L., Zhang, D. \& Pan, Q. (2009). Object separation by polarimetric and spectral imagery fusion. Computer Vision and Image Understanding 113, 855-866.

\section{ADDITIONAL READING SECTION}

Born, M. \& Wolf, E. (1999). Principles of Optics: Electromagnetic Theory of Propagation, Interference and Diffraction of Light (7th Edition). Cambridge University Press.

Cox, W. C., Hughes, B. L. \& Muth, J. F. (2009). A polarization shift-keying system for underwater optical communications. In OCEANS 2009, MTS/IEEE Biloxi - Marine Technology for Our Future: Global and Local Challenges.

Cronin, T. W., Shashar, N., Caldwell, R. L., Marshall, J., Cheroske, A. G. \& Chiou, T.-H. (2003). Polarization vision and its role in biological signaling. Integrative and Comparative Biology 43 (4), 549-558.

Furukawa, T. \& Sakamoto, K. (2010). Eye contact communication system between mobile robots using invisible code display. In H. Yang, R. Malaka, J. Hoshino \& J. Han (ed.),Entertainment Computing ICEC 2010, Vol. 6243 (pp. 468-471). Springer Berlin / Heidelberg.

Giakos, G. C. (2006). Multifusion, multispectral, optical polarimetric imaging sensing principles. IEEE Transactions on Instrumentation and Measurement 55, 1628-1633.

Goldstein, D. (2003). Polarized Light, Revised and Expanded (Optical Science and Engineering). CRC.

Horváth, G. \& Varjú, D. (2004). Polarized light in animal vision: Polarization patterns in nature. Springer.

Können, G. P. (1985). Polarized light in nature. Cambridge University Press.

Lambrinos, D., Möller, R., Labhart, T., Pfeifer, R. \& Wehner, R. (2000). A mobile robot employing insect strategies for navigation. Robotics and Autonomous Systems 30, 39-64.

Lerner, A., Sabbah, S., Erlick, C. \& Shashar, N. (2010). Navigation by light polarization in clear and turbid waters. Philosophical Transactions of The Royal Society Biological Sciences 366, 671-679. 
Li, Y. \& Wang, S. (2010). Underwater object detection technology based on polarization image fusion. In 5th International Symposium on Advanced Optical Manufacturing and Testing Technologies: Optoelectronic Materials and Devices for Detector, Imager, Display, and Energy Conversion Technology, Proc. of SPIE.

Miyazaki, D., Ammar, M., Kawakami, R. \& Ikeuchi, K. (2009). Estimating sunlight polarization using a fish-eye lens. IPSJ Transactions on Computer Vision and Applications 1, 288-300.

Schechner, Y. Y., Narasimhan, S. G. \& Nayar, S. K. (2008). Polarization-based vision through haze. In Proceeding of ACM SIGGRAPH ASIA, Vol. 42.

Shabayek, A. E. R. (2010). Non-central catadioptric sensors auto-calibration. LAP Lambert Academic Publishing.

Tyo, J. S. (1998). Optimum linear combination strategy for an N-channel polarization-sensitive imaging or vision system. Journal of Optical Society 15, 359-366.

Yemelyanov, K. M., Lin, S.-S., Luis, W. Q., Pugh, J. E. N. \& Engheta, N. (2003). Bio-inspired display of polarization information using selected visual cues. In Proceedings of SPIE - The International Society for Optical Engineering 5158.

Zhang, Z. Y., Cheng, H. F., Chen, Z. H. \& Zheng, W. W. (2009a). Analysis of polarimetric characteristics of camouflage targets. In Proceedings of SPIE 4th International Symposium on Advanced Optical Manufacturing and Testing Technologies: Optical Test and Measurement Technology and Equipment, Vol. 7283.

Zhao, Y.-Q., Gong, P. \& Pan, Q. (2008). Object detection by Spectropolarimeteric Imagery Fusion. IEEE Transactions on Geoscience and Remote Sensing 46, 3337-3345.

\section{KEY TERMS \& DEFINITIONS}

Polarization: It is the phenomenon that describes the oscillations orientation of waves which are restricted in direction.

Orientation: The ability to decide on correct direction.

Navigation: The ability to decide on correct route.

Communication: The ability to exchange a simple message.

Camouflage: The ability to hide.

Polarimetic camera: A camera that is able to capture polarization characteristics represented in intensity images.

Omni-directional image: An image which has a wide extent of the surrounding environment.

Catadioptric camera: A combination of mirror and lens to obtain omni-directional images up to $360^{\circ}$. 
Omni-directional polarization vision: The ability to perceive the surrounding environment, up to $360^{\circ}$, in terms of its polarimetric values.

Omni-spectro-polarization vision: The ability to perceive the surrounding environment, up to $360^{\circ}$, in terms of its spectral and polarimetric values. 